\title{
A microfluidic system for dynamic yeast cell imaging
}

\author{
Philip J. Lee, ${ }^{1}$ Noah C. Helman, ${ }^{2}$ Wendell A. Lim, ${ }^{2}$ and Paul J. Hung ${ }^{1}$ \\ ${ }^{1}$ CellASIC Corporation, San Leandro and 2 Department of Cellular and Molecular \\ Pharmacology, UC San Francisco, San Francisco, CA, USA
}

BioTechniques 44:91-95 (January 2008)

doi 10.2144/000112673

The investigation of cellular processes and gene regulatory networks within living cells requires the development of improved technology for dynamic, single cell imaging. Here, we demonstrate a microfluidic system capable of mechanical trapping of yeast cells with continuous flow and flow switching capability during time-lapse high magnification fluorescence imaging. The novel functionality of the system was validated by observing the response of pheromone-induced expression of GFP in Saccharomyces cerevisiae.

\section{INTRODUCTION}

Cells are dynamic systems that respond to certain changes in their environment, but most studies of this response lack the ability to apply precise time-varying stimuli. Recent interest in variation of the response among an isogenic population highlights the need for experimental systems that monitor single cells (1). In order to properly characterize a time-dependent cellular system, an experimental platform for subjecting a clonal population of cells to time-varying stimuli and following the individual cells via time-lapse microscopy would be a valuable tool.

The use of microfluidic engineering methods provides unique capabilities for live cell analysis (2-4). By implementing continuous flow systems, a number of researchers have been able to create steady state environments for long-term culture and monitoring of bacteria and yeast cells (5-7). For example, Hasty and colleagues designed a "Tesla microchemostat" for improved gene expression monitoring in Saccharomyces cerevisiae using fluorescence microscopy (8). This work highlighted the importance of maintaining precise cell localization for long-term imaging studies. In this current manuscript, we describe a fully functional system for yeast cell imaging experiments with flow control and flow switching capability. The unique features of this system are: $(i)$ the use of elastic chambers to mechanically trap yeast cells in a uniform focal plane; (ii) the design of the flow resistance network to ensure robust and repeatable performance; (iii) the ability to rapidly change the solution around the cells during imaging without causing physical displacement of the cells; and (iv) the integration of the microfluidics with a 96-well plate and a customized pneumatic flow controller for ease of operation.

The major goal of this work was to create a microfluidic system for widespread use among the biology community for live cell imaging of yeast cells. While the concept of a microfluidic flow chamber has been demonstrated on multiple occasions, there is currently a lack of commercially available products that enable robust live cell experimentation with the features listed above. To reach this goal, we developed key engineering innovations that are not currently available in the microfluidics field. The end result is a substantial improvement in "ease-of-use" as well as functional reliability. For example, we based the microfluidics on a standard 96-well plate format that eliminates the use of wetted tubing and fluidic connections. This circumvents the common problems associated with microfluidic devices, such as air bubble introduction, tedious setup times, and limited flow configurations. This format also enabled the implementation of a customized flow control system to enable experiment setup at a fraction of the time and cost of existing methods (9).

\section{MATERIALS AND METHODS}

\section{Microfluidic Design}

The microfluidic device was designed to operate with the flow control system available from CellASIC Corporation (San Leandro, CA, USA). The microfluidic chambers are also commercially available for laboratory use from the same company. These were designed to be operated similarly to a standard microtiter dish. The operation steps include: $(i)$ pipet up to $300 \mu \mathrm{L}$ of cells and exposure solutions to the inlet wells; (ii) attach the flow control system to the microfluidic plate; and (iii) place on an inverted microscope and perform time-lapse experimentation. The construction of the device consists of an open bottom 96-well plate adhered to an acrylic backed microfluidic device molded from elastomer. The bottom surface of the microfluidics is then bonded to a glass cover slide to allow for high magnification microscopy.

The microfluidic layout is depicted in Figure 1A, and consists of two flow inlets, two cell inlets, an imaging area, and an outlet. Each circular region corresponds to a single well on a standard 96-well plate. The "trapping site" is enlarged in Figure $1 \mathrm{~B}$, and represents the region of interest for cell microscopy. The upstream channels are not depicted in detail, but are designed to provide the correct resistance ratio for device operation as explained in the Results and Discussion section. Each microfluidic plate allowed observation of two different yeast strains in parallel, with simultaneous exposure to one of two different flow solutions. The fluidic channels were fabricated by molding polydimethylsiloxane (PDMS) elastomer on a microfabricated mold as previously described (10).

The yeast cell imaging region consisted of a series of elastic trapping sites $160 \mu \mathrm{m}$ in width and $60 \mu \mathrm{m}$ in length (Figure 1B). Cells were loaded from the corresponding inlet channel, and exposed to the flow solutions from 
A

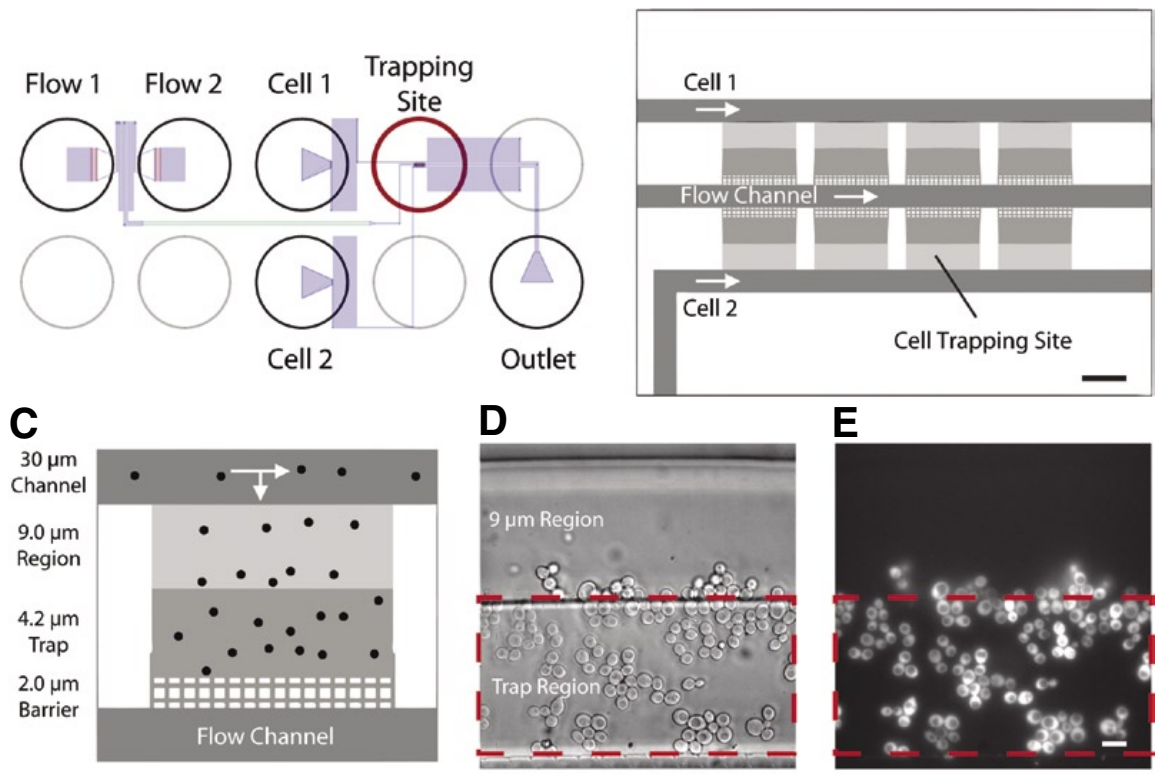

Figure 1. Microfluidic circuit for yeast cell imaging. (A) The single fluidic unit was formatted to cover 6 wells of a 96-well plate. This layout allowed for two reagent solutions, two cell types, cell microscopy, and a flow outlet. The outline of the wells is superimposed on the microfluidic channels. (B) The cell trapping area consists of eight trapping sites where yeast cells are mechanically held for time-lapse imaging. A central flow channel delivers the flow solutions simultaneously to the two different cell types. Scale bar represents $100 \mu \mathrm{m}$. (C) Each trapping site is separated from the flow channel by a 2 $\mu \mathrm{m}$ barrier to prevent cells from passing through while enabling rapid solution transport. A 4.2 $\mu \mathrm{m}$ elastic pad was designed to clamp yeast cells against an underlying glass slide for time-lapse imaging. (D) Brightfield and (E) fluorescence images of GFP-expressing Saccharomyces cerevisiae cells trapped in the microfluidic site. Note the homogeneous focal plane in the $4.2 \mu \mathrm{m}$ region (bottom half) as compared with the $9.0 \mu \mathrm{m}$ region (top half). Scale bar represents $10 \mu \mathrm{m}$. the opposite side. The flow path across the cells during solution exposure is opposite than that of cell loading, helping to wash out cells that weren't trapped and prevent channel clogging. A small cross-section microfluidic barrier was patterned as described in previous work (11) to separate the cells from the flow channel while enabling rapid mass transport (Figure 1C). This consisted of a grid-like network of shallow 2 $\mu \mathrm{m}$ channels that would act as a cell filter. The "cross-wired" appearance was designed to prevent clogging of the flow path as the cells were filtered. Additionally, a $9 \mu \mathrm{m}$ region upstream of the trap limited cell numbers at the edge of the trap site, reducing the chance of a bottleneck. Cell loading was achieved by pressurizing the cell inlet wells to drive the cells into the imaging region. The cells trapped in the $4.2 \mu \mathrm{m}$ region were maintained in a fixed $x, y, z$ position during time-lapse imaging using brightfield and fluorescence microscopy (Figure 1, D and E). This design solved the major limitations of existing flow systems for yeast microscopy by maintaining live cells in focus in a fixed location while enabling continuous and uniform solution exchange. Extending this architecture to accommodate larger cells will require simply designing a correspondingly larger trapping chamber height.
The height of the cell trapping chamber was experimentally measured using polystyrene microspheres (NIST Traceable Particle Size Standards, Polysciences, Warrington, PA, USA). Three bead sizes were tested $(4.17 \pm$ $0.03 \mu \mathrm{m}, 5.34 \pm 0.04 \mu \mathrm{m}$, and $5.80 \pm$ $0.45 \mu \mathrm{m})$ to determine the pressure required to load each bead size. The cross-sectional height of the trapping region was also measured at 1.0 micron intervals with a resolution of $<10$ nanometers using a surface profilometer (Alpha-Step IQ, KLA Tencor, San Jose, CA, USA).

\section{Yeast Cell Analysis}

The function of the microfluidic yeast cell imaging system was demonstrated by observing the expression of GFP by the Fus1 promoter in response to $\alpha$-factor stimulation in standard a-type haploid cells [SO992 (12)] with far1 $\Delta$, bar1::KanR, mfa2:: $\left.\mathrm{P}_{\mathrm{FUS} 1}-\mathrm{GFP}\right)$. During this experiment, cells were arrested in $\mathrm{S}$ phase using $200 \mathrm{mM}$ hydroxyurea for $2 \mathrm{~h}$ before being loaded into the microfluidic chamber at an appropriate density. Trapped cells were exposed to a 40 min pulse of $2 \mu \mathrm{M} \alpha$-factor in synthetic complete medium (SC). From $40 \mathrm{~min}$ to $120 \mathrm{~min}$, the cells were washed with $\mathrm{SC}$, at which point the fluid flow was switched back to $2 \mu \mathrm{M} \alpha$-factor for the remainder of the experiment. Since the trapped yeast cells remained fixed in space during the flow switching, our custom-written software could analyze the fluorescence from individual cells tracked through the entire time-lapse movie. Imaging was performed with an automated inverted Nikon microscope with Perfect Focus using a $60 \times$ oil immersion lens (Nikon, Tokyo, Japan). Postprocessing of the images was performed by custom software written in MATLAB (The MathWorks, Natick, MA, USA), which identified each cell in the frame using the brightfield images, determined the cell boundaries, and measured the GFP intensity from the fluorescence channel. For this experiment 186 cells were simultaneously tracked and the mean and standard deviation (SD) computed for each 5 min time point.

\section{RESULTS AND DISCUSSION}

The microtiter plate format of the microfluidic devices facilitated operations such as liquid dispensing, environment control, and imaging on an unmodified inverted microscope. This flexible layout is amenable to future improvement, such as increased throughput (e.g., 384-well spacing), 
A

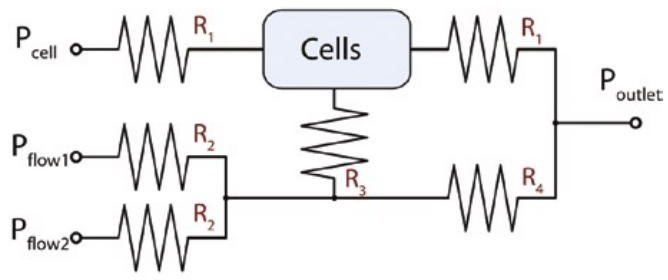

B
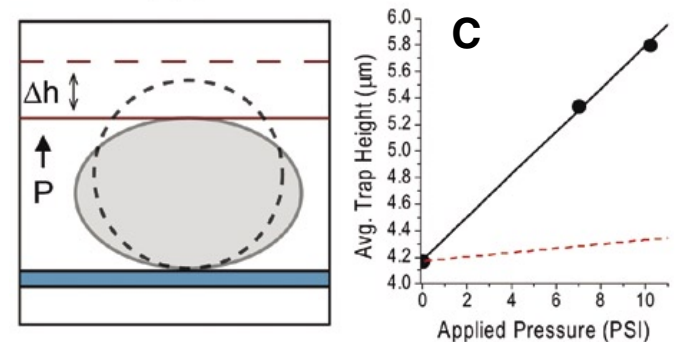

D
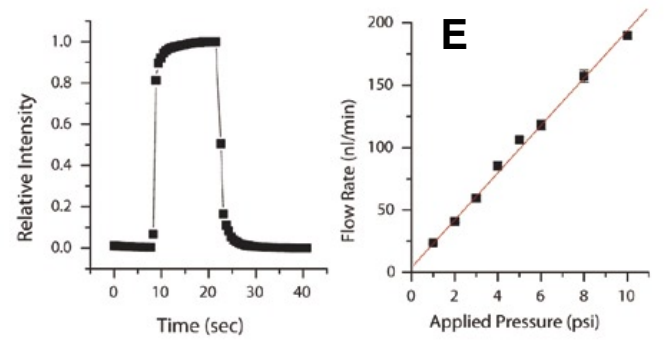

Figure 2. Microfluidic dynamics. (A) Resistance network of the microfluidic flow unit. The ratio of resistances $\left(\mathrm{R}_{1}: \mathrm{R}_{2}: \mathrm{R}_{3}: \mathrm{R}_{4}\right)$ was set at 1:10:1:0.1. The layout of the solution wells allowed each inlet port $\left(\mathrm{P}_{\text {cell }}, \mathrm{P}_{\text {flow1 }}, \mathrm{P}_{\text {flow } 2}\right)$ to be independently controlled with pneumatic pressure. (B) The elastic cell trap was designed with an initial height of $4.2 \mu \mathrm{m}$ and expanded under pressure. When the cell loading port was pressurized, this caused the flow of cells into the expanded trap (dotted lines). Once this pressure was released, the ceiling returned to its original height, immobilizing the yeast cells. (C) Channel height as a function of applied pressure to the cell loading port (-) and the flow inlet ports (---). Values were measured with $4.2,5.3$, and $5.8 \mu \mathrm{m}$ microspheres and fit to a mechanical model. (D) The design of the two solution inlet ports allowed rapid flow switching and (E) flow rate control without any displacement of the imaged cells.

temperature control (via custom or off-the shelf plate heaters), automated liquid dispensing, and adaptation for high-content analysis. The main focus of this article is to describe how a microfluidic flow chamber was designed and implemented for use in dynamic cell imaging of yeast.

The central innovation of the microfluidic design was the tuning of fluidic resistances through the microfluidic network to maximize performance of cell loading and flow switching (Figure 2A). At low Reynolds number ( $R e$ $<0.1$ ), fluid flow is highly laminar and follows the "Ohm's Law" relation $Q=$ $\Delta P / R$, where $Q$ is the flow rate, $\Delta P$ is the pressure drop, and $R$ is the fluidic resistance. Furthermore, the value of the fluidic resistance through each flow path was designed to tolerate intrinsic pressure variations (room vibration, thermal fluctuation, gravity driven flow) to eliminate unwanted convective transport. We took advantage of these properties to create a valveless design for on-chip fluid switching. This was accomplished by placing 100-fold higher fluidic resistance upstream $\left(\mathrm{R}_{2}\right)$ of the switch branch compared with flow to the downstream outlet $\left(R_{4}\right)$, therefore making flow back through the opposite inlet extremely unfavorable.
For example, if flow inlet 1 is pressurized $\left(\mathrm{P}_{\text {flow } 1}\right)$ at 5 psi, then $99 \%$ of the pressure drop is across $\mathrm{R}_{2}$, which means by the time the liquid reaches the main channel, the local pressure is only $0.3 \%$ above atmospheric. Due to the high resistance ratio between backflow observed only diffusive mixing into the upstream channel. During a $3 \mathrm{~h}$ experiment with continuous flow at 5 psi, this backflow was less than $10 \%$ of the upstream channel volume. Since mixing is slow in the laminar regime, this "contaminated" plug (of $<10 \mathrm{nl}$ ) was washed out within $1 \mathrm{~s}$ of turning on the corresponding switch. In similar experiments, continuous flows of over $10 \mathrm{~h}$ were reliably performed such that the back mixing did not extend beyond the upstream channel volume (and never contacted the reservoir well).

Increasing the local hydrostatic pressure caused the height of the elastic channel to increase such that yeast cells could be transported into the mechanical cell trapping site (Figure 2B). The resting height of the channel was $4.2 \mu \mathrm{m}$, and could expand to $6 \mu \mathrm{m}$ when pressurized (the typical haploid yeast cell in our experiment was $5 \pm 0.5$ $\mu \mathrm{m}$ in diameter). The channel expansion was measured experimentally using (up to $\left.\mathrm{P}_{\text {flow2 }}\right)$ and the outlet $\left(\mathrm{P}_{\text {outlet }}\right)$, we microspheres of defined sizes (Figure 2C). The trap size responded linearly with applied pressure $\left(\mathrm{R}^{2}=0.998\right)$ as predicted by a previously published model for low aspect ratio PDMS channels (13). The channel expansion in the medium flow (reverse) direction was below the limit of detection, but is expected to be 10-fold smaller due to the reduced hydrostatic pressure as a result of the higher upstream resistance. This ensured that after the cells were loaded, pressure changes caused by switching of solutions would not lead to any cell displacement during imaging.

To demonstrate system operation, wild-type $S$. cerevisiae was loaded into the microfluidic chamber at 3 psi for $30 \mathrm{~s}$ (see Supplementary Movie 1 available online at www.BioTechniques.com). A wash step of buffer solution (from Flow 1) at 5 psi for $1 \mathrm{~min}$ removed nontrapped cells. The flow profile of a fluorescent dye $(250 \mu \mathrm{g} / \mathrm{mL}$ Texas Red conjugated dextran, MW 10,000) was traced through the cell trapping site, showing the ability to generate flow profiles with complete medium turnover within a few seconds (Figure 2D; see Supplementary Movie 2). The liquid flow rate between 25 and $200 \mathrm{nl} / \mathrm{min}$ was linear over the operating range with respect to the applied pressure (Figure $2 \mathrm{E})$. This allows the liquid volume in the inlet reservoir $(300 \mu \mathrm{L})$ to sustain continuous perfusion for long-term studies including, for instance, experiments following multiple cell division cycles (see Supplementary Movie 3). Even after 3 days of cell division, flow switching remained unimpeded. This is believed to be due to the lack of "dead space" where cells could accumulate. As the cells overgrew the trapping regions, the continuous flow would carry them downstream into the outlet wells (see Supplementary Movie 4).

The reliability of device fabrication was tested over 30 replications of a single mold. Since the channel heights were defined by silicon and crosslinked photoresist (SU-8) and subsequently coated with Teflon, the molding process had no noticeable effect on the trap size from batch to batch. From measurements over 30 consecutive molding cycles, there was less than a $50 \mathrm{~nm}$ change in the cell trap height. 
The cross-section of the trap area also showed a very uniform profile, with a SD across the trapping sites of $0.1 \%$ $(0.004 \mu \mathrm{m})$ and a maximum deviation of $0.4 \%(0.02 \mu \mathrm{m})$.

The ability to image individual yeast cells during flow switching was tested with an inducible GFP expressing cell type over a period of hours (Figure 3; Supplementary Movie 5). In this experiment, the yeast strain responds to $\alpha$-factor pheromone by transcribing the enhanced green fluorescent protein (EGFP) gene from the $\mathrm{P}_{\mathrm{FUS} 1}$ promoter. By pulsing the cells with pheromone and washing out, we were able to observe the response of the population to this stimulus. The delay between the stimulus and response is a convolution of the delay of the information processing capability of the system (G-protein coupled receptor, MAPK cascade, transcription factor activation) and the transcription, translation, folding, and chromophore maturation of GFP. The large variation in response between all the cells that were measured is likely a combination of effects from stochastic noise in the signaling pathway and the transcription/translation machinery. Cell cycle effects are less likely as all cells were arrested in $200 \mathrm{mM}$ hydroxyurea for $2 \mathrm{~h}$ prior to the experiment as well as during the experiment.

This work describes a promising new platform for performing dynamic live cell studies on yeast. Since $S$. cerevisiae is a nonadherent cell type, it is not trivial to image cells over time when exposed to flow. Current methods such as concanavalin A coating and agar pad culture do not maintain all the cells in a uniform focal plane. Furthermore, while other microfluidic chambers have been published for cell imaging, it is important to emphasize the commercial availability of this current platform. Along these lines, key innovations have been developed to ensure that the system is costcompetitive and reliable for everyday experimentation. The simple design and ease of use of this current microfluidic system makes it applicable to a wide range of studies focusing on the dynamical behavior of single cells, such as gene expression,
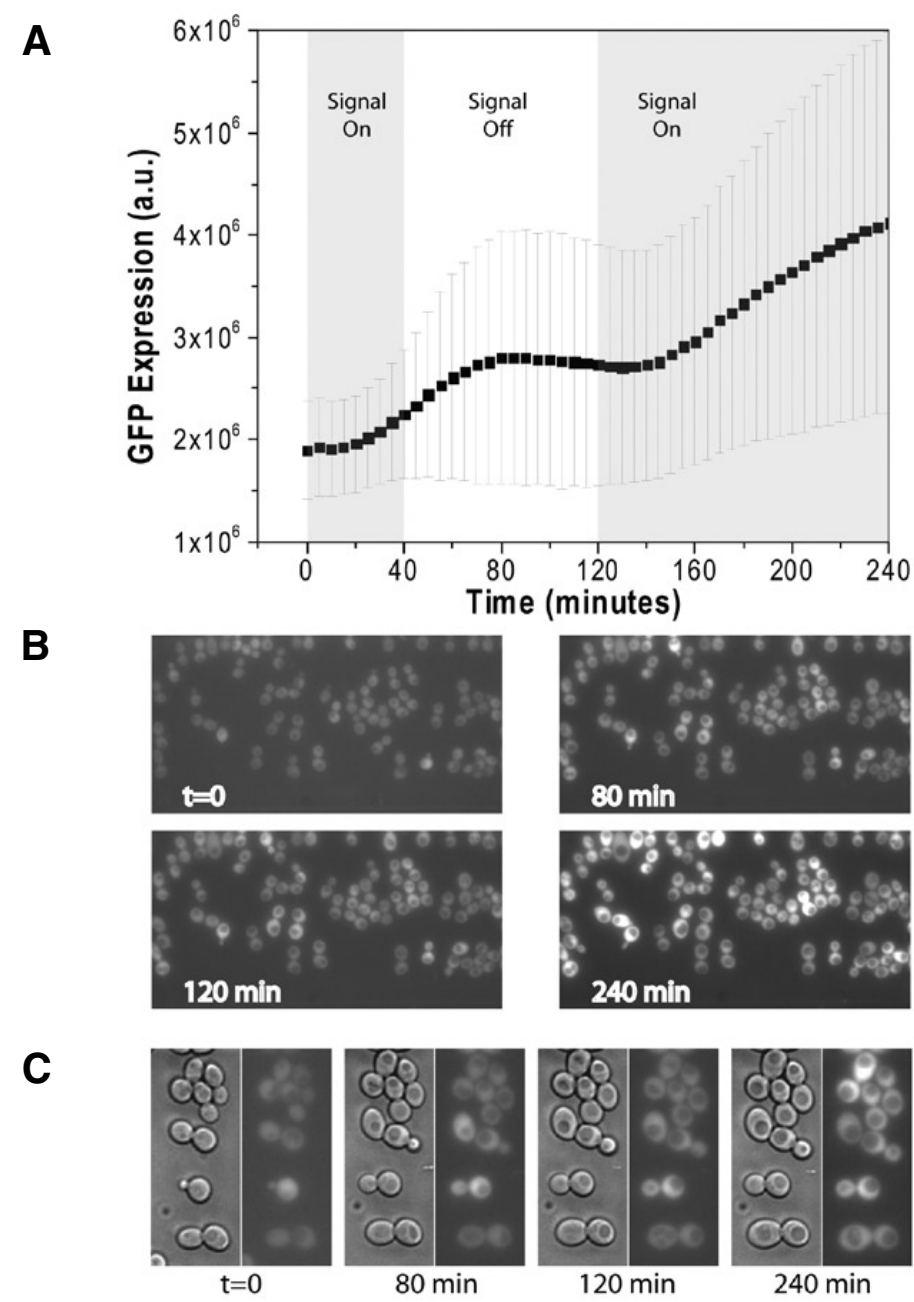

$120 \mathrm{~min}$

$240 \mathrm{~min}$

Figure 3. Time-lapse cell imaging. (A) $\mathrm{P}_{\mathrm{FUS1}}-\mathrm{GFP}$ expression in Saccharomyces cerevisiae cells in response to solution switching in the microfluidic device over $4 \mathrm{~h}$. The mean and SD of GFP intensity was plotted for all 186 cells in a single trap region. (B) Microscope images from a single trapping site during the same time course. (C) Brightfield and GFP images depicting cell growth without physical displacement during long-term flow.

protein translocation and localization, cell division, and cell signaling. It is also possible to expand the microfluidic device to conduct multiplexed parallel flow experiments, such that multiple cell types or flow conditions are compared side-by-side. The continued adoption of microfluidic methods by cell biologists will enable significantly improved research tools to investigate the complex behaviors of living cells.

\section{ACKNOWLEDGMENTS}

N.H. is supported by Postdoctoral Fellowship PF-06-194-01-TBE from the American Cancer Society.

\section{COMPETING INTERESTS STATEMENT}

The authors declare no competing interests.

\section{REFERENCES}

1. Colman-Lerner, A., A. Gordon, E. Serra, T. Chin, O. Resnekov, D. Endy, C.G. Pesce, and R. Brent. 2005. Regulated cell-to-cell variation in a cell-fate decision system. Nature 437:699-706. 
2. El-Ali, J., P.K. Sorger, and K.F. Jensen. 2006. Cells on chips. Nature 442:403-411.

3. Di Carlo, D. and L.P. Lee. 2006. Dynamic single-cell analysis for quantitative biology. Anal. Chem. 78:7918-7925.

4. Breslauer, D.N., P.J. Lee, and L.P. Lee. 2006. Microfluidics-based systems biology. Mol. Biosyst. 2:97-112.

5. Balagaddé, F.K., L. You, C.L. Hansen, F.H. Arnold, and S.R. Quake. 2005. Longterm monitoring of bacteria undergoing programmed population control in a microchemostat. Science 309:137-140.

6. Groisman, A., C. Lobo, H. Cho, J.K. Campbell, Y.S. Dufour, A.M. Stevens, and A. Levchenko. 2005. A microfluidic chemostat for experiments with bacterial and yeast cells. Nat. Methods 2:685-689.

7. Paliwal, S., P.A. Iglesias, K. Campbell, Z. Hilioti, A. Groisman, and A. Levchenko. 2007. MAPK-mediated bimodal gene expression and adaptive gradient sensing in yeast. Nature 446:46-51.

8. Cookson, S., N. Ostroff, W.L. Pang, D. Volfson and J. Hasty. 2005. Monitoring dynamics of single-cell gene expression over multiple cell cycles. Mol Syst Biol 1:2005.0024

9. Lee, P.J., N. Ghorashian, T.A. Gaige, and P.J. Hung. Microfluidic system for automated cell-based assays. J. Assoc. Lab. Autom. 12:363-367.

10. Hung, P.J., P.J. Lee, P. Sabounchi, N. Aghdam, R. Lin, and L.P. Lee. 2005. A novel high aspect ratio microfluidic design to provide a stable and uniform microenvironment for cell growth in a high throughput mammalian cell culture array. Lab Chip 5:44-48.

11. Lee, P.J., P.J. Hung, and L.P. Lee. 2007. An artificial liver sinusoid with a microfluidic endothelial-like barrier for primary hepatocyte culture. Biotechnol Bioeng. 97:1340-1346

12. O'Rourke, S.M. and I. Herskowitz. 2002. A third osmosensing branch in Saccharomyces cerevisiae requires the Msb2 protein and functions in parallel with the Sho1 branch. Mol. Cell. Biol. 22:4739-4749.

13. Gervais, T., J. El-Ali, A. Gunther, and K.F. Jensen. 2006. Flow-induced deformation of shallow microfluidic channels. Lab Chip 6:500-507.

Received 22 June 2007; accepted 10 October 2007.

Address correspondence to Philip J Lee, CellASIC Corporation, 2551 Merced St., San Leandro, CA, 94577, USA. e-mail: pjlee@cellasic.com

To purchase reprints of this article, contact: Reprints@BioTechniques.com 